\title{
Isotermas de dessorção de folhas in natura de juazeiro e mororó ${ }^{1}$
}

\author{
Francinalva Cordeiro de Sousa ${ }^{2}$, Ana Paula Trindade Rocha ${ }^{2}$, \\ Josivanda Palmeira Gomes ${ }^{2}$, Jorge Jacó Alves Martins², Joabis Nobre Martins ${ }^{2}$
}

\begin{abstract}
Desorption isotherms of in natura leaves of Ziziphus joazeiro Mart. and Bauhinia cheilantha

The Brazilian culture has a great appreciation for the use of medicinal plants for curing diseases. The drying process is very important to assure the quality and stability of these species. This study aimed at obtaining the desorption isotherms of in natura leaves of Ziziphus joazeiro Mart. and Bauhinia cheilantha and adjust the experimental data to different mathematical models. The isotherms were obtained at three temperatures $\left(20^{\circ} \mathrm{C}, 30^{\circ} \mathrm{C}\right.$ and $40^{\circ} \mathrm{C}$ ), under desorption conditions, and the experimental data adjusted to three models (GAB, Oswin and Peleg) by nonlinear regression. The coefficient of determination and the mean percentage deviation between the experimental and predicted values were calculated for each curve, in order to evaluate the best-fit model. According to the statistical parameters of analysis, the Peleg model was the one that better described the hygroscopicity of in natura leaves of Ziziphus joazeiro Mart. and Bauhinia cheilantha.
\end{abstract}

KEY-WORDS: Ziziphus joazeiro Mart.; Bauhinia cheilantha; medicinal plants; mathematical modeling.

\section{INTRODUÇÃO}

A cultura brasileira tem grande apreço pela utilização de plantas medicinais, na cura de doenças. A comercialização dessas plantas é realizada na forma dessecada, o que torna a secagem uma das etapas que assume grande importância, sendo muito utilizada no processo para assegurar a qualidade e a estabilidade desse material.

Dentre as espécies nativas do semiárido nordestino, 119 são utilizadas como plantas medicinais (Agra et al. 2007), com destaque para o juazeiro e o mororó. Essas duas espécies do bioma Caatinga vêm se destacando pelo seu enorme potencial econômico e medicinal. Comercialmente, o juazeiro é utilizado

\section{RESUMO}

A cultura brasileira tem grande apreço pela utilização de plantas medicinais, na cura de doenças. O processo de secagem é de grande importância para assegurar a qualidade e a estabilidade dessas espécies. O presente estudo objetivou obter as isotermas de dessorção de folhas in natura de juazeiro (Ziziphus joazeiro Mart.) e mororó (Bauhinia cheilantha) e ajustar os dados experimentais a diferentes modelos matemáticos. As isotermas foram obtidas em três temperaturas $\left(20^{\circ} \mathrm{C}, 30^{\circ} \mathrm{C}\right.$ e $\left.40^{\circ} \mathrm{C}\right)$, em condições de dessorção, e os dados experimentais ajustados a três modelos (GAB, Oswin e Peleg), por meio de regressão não linear. $\mathrm{O}$ coeficiente de determinação e o desvio percentual médio entre os valores experimentais e os valores preditos foram calculados para cada curva, visando a verificar o modelo que apresentou melhor ajuste. Segundo os parâmetros estatísticos de análise, o modelo de Peleg foi o que descreveu melhor a higroscopicidade das folhas in natura de juazeiro e mororó.

PALAVRAS-CHAVE: Ziziphus joazeiro Mart.; Bauhinia cheilantha; plantas medicinais; modelagem matemática.

por indústrias farmacêuticas, na fabricação de cosméticos, creme dental e xampus anticaspa. Já o mororó é reconhecidamente utilizado no combate à diabetes (Sousa et al. 2013).

O teor de água de qualquer produto, quando em equilíbrio com o ambiente de armazenamento, é chamado de conteúdo de umidade de equilíbrio, ou equilíbrio higroscópico. O conteúdo de umidade de equilíbrio avalia a perda ou ganho de água sob determinada condição de temperatura e umidade relativa, relacionando-se diretamente com os processos de secagem e armazenagem de produtos agrícolas. O conteúdo de umidade de equilíbrio é alcançado quando a pressão parcial de vapor de água no produto iguala-se à pressão parcial de vapor do ar que o envolve (Costa

1. Trabalho recebido em set./2013 e aceito para publicação em ago./2014 (nº registro: PAT 26374).

2. Universidade Federal de Campina Grande (UFCG), Centro de Tecnologia e Recursos Naturais, Campina Grande, PB, Brasil. E-mails: francis_nalva@yahoo.com.br, ana_trindade@yahoo.com.br,josivanda@gmail.com,jaco-m@hotmail.com, martinsjnta@gmail.com. 
2010). O teor de umidade de equilíbrio dos produtos biológicos, por meio da determinação de isotermas de secagem, está relacionado ao dimensionamento de secadores.

Segundo Resende et al. (2006), os produtos agrícolas interagem com o ambiente, cedendo ou absorvendo água, com tendência ao equilíbrio entre o seu teor de água e a umidade do ambiente. $\mathrm{Na}$ condição de equilíbrio, a relação entre a umidade do produto, umidade relativa do ar e temperatura pode ser expressa por equações matemáticas, cuja denominação é conhecida por isotermas de sorção, ou curvas de equilíbrio higroscópico (Ferreira et al. 2011).

A qualidade do material biológico pode ser preservada mediante estudo de parâmetros como secagem e armazenamento. $\mathrm{O}$ conhecimento sobre a umidade de equilíbrio desses materiais é bastante importante, pois define as condições adequadas para o armazenamento de drogas vegetais. Por meio das curvas de sorção, que relacionam a umidade de equilíbrio do produto e a umidade relativa, com temperatura constante, é possível determinar o teor de água final necessário à estabilização do material.

Este trabalho objetivou estudar a influência da temperatura nas isotermas de dessorção de juazeiro e mororó, visando a obter o modelo matemático que melhor satisfaça às condições avaliadas.

\section{MATERIAL E MÉTODOS}

Os experimentos para obtenção dos valores da umidade de equilíbrio do produto foram realizados no Laboratório de Armazenamento e Processamento de Produtos Agrícolas da Unidade Acadêmica de Engenharia Agrícola da Universidade Federal de Campina Grande (PB). Foram utilizadas, como matéria-prima, folhas in natura de juazeiro (Ziziphus joazeiro Mart.) e mororó (Bauhinia cheilantha), coletadas no Câmpus da unidade de Campina Grande, entre março e abril de 2012.

As isotermas de dessorção de umidade das folhas de juazeiro e mororó foram determinadas em três diferentes temperaturas $\left(20^{\circ} \mathrm{C}, 30^{\circ} \mathrm{C}\right.$ e $\left.40^{\circ} \mathrm{C}\right)$, utilizando-se o método estático-indireto (Capriste \& Rotstein 1982).

A atividade de água $\left(\mathrm{a}_{\mathrm{w}}\right)$ das amostras de folhas in natura, para as três diferentes temperaturas, foi determinada em analisador de atividade de água Aqualab 3TE (Decagon). Ao iniciar os experimentos, foram pesadas, em balança analítica com precisão de $0,0001 \mathrm{~g}$, aproximadamente $1,000 \mathrm{~g}$ de folhas frescas de juazeiro e mororó, utilizando-se as cápsulas de polietileno que acompanham o equipamento. Em seguida, as mesmas foram mantidas em estufa com circulação forçada de ar, a $60^{\circ} \mathrm{C}$, por 15 minutos. Após esse período, as amostras foram transferidas para um dessecador, para equilibrar a temperatura da amostra com a do equipamento Aqualab, a fim de que a leitura fosse realizada de forma correta, sem que houvesse interferência do ambiente. Logo após, as amostras foram colocadas, uma de cada vez, no equipamento Aqualab, para realizar as leituras de atividade de água e temperatura.

$\mathrm{O}$ registro da atividade de água foi realizado mediante o equilíbrio atingido entre a amostra e o equipamento, evidenciado pela estabilização da leitura. Na sequência, as amostras foram pesadas em balança analítica, procedendo-se à leitura de 5 em 5 minutos, processo que se repetiu até que a última leitura apresentasse valor igual ou maior que a penúltima, possibilitando, dessa forma, a construção das isotermas de dessorção. Esse procedimento foi realizado para as amostras em triplicata, nas três temperaturas. Após as amostras alcançarem valores de atividades de água próximos a 0,1 , essas foram levadas a estufa, para determinação da massa seca e, consequentemente, da umidade de equilíbrio.

A umidade de equilíbrio, em base seca, foi determinada pela relação entre a massa de água e a massa seca das amostras:

$$
\mathrm{X}_{\mathrm{eq}}=\frac{\mathrm{m}_{\mathrm{eq}}-\mathrm{m}_{\mathrm{s}}}{\mathrm{m}_{\mathrm{s}}} 100
$$

em que $X_{\text {eq }}=$ umidade de equilíbrio (\% base seca); $\mathrm{m}_{\mathrm{eq}}=$ massa da amostra no equilíbrio $(\mathrm{g}) ; \mathrm{m}_{\mathrm{s}}=$ massa seca da amostra (g).

Utilizaram-se, para o ajuste matemático das isotermas de dessorção de teor de água das amostras de folhas, os modelos descritos a seguir, por meio de regressão não linear, pelo método Quasi-Newton, empregando-se o programa computacional Statistica $5.0^{\circledR}$ :

GAB: $X_{e q}=\frac{X_{m} \mathrm{CKa}_{w}}{\left(1-\mathrm{Ka}_{\mathrm{w}}\right)\left(1-\mathrm{Ka}_{\mathrm{w}}+\mathrm{CKa}_{\mathrm{w}}\right)}$

Peleg: $\mathrm{X}_{\mathrm{eq}}=\mathrm{k}_{1} \mathrm{a}_{\mathrm{w}}{ }^{\mathrm{n} 1} \mathrm{k}_{2} \mathrm{a}_{\mathrm{w}}{ }^{\mathrm{n} 2}$

Oswin: $\mathrm{X}_{\mathrm{eq}}=\mathrm{a}\left(\frac{\mathrm{a}_{\mathrm{w}}}{\left(1-\mathrm{a}_{\mathrm{w}}\right)}\right)^{\mathrm{b}}$ 
em que $\mathrm{X}_{\mathrm{eq}}=$ umidade de equilíbrio; $\mathrm{a}_{\mathrm{w}}=$ atividade de água; $\mathrm{X}_{\mathrm{m}}=$ umidade na monocamada molecular; $\mathrm{C}$, $\mathrm{K}$, $\mathrm{a}$ e $\mathrm{b}=$ parâmetros que dependem da temperatura e da natureza do produto; $\mathrm{K}_{1}, \mathrm{~K}_{2}, \mathrm{n}_{1}$ e $\mathrm{n}_{2}=$ constantes da equação.

Os critérios usados para determinação do melhor ajuste dos modelos aos dados experimentais foram o coeficiente de determinação $\left(\mathrm{R}^{2}\right)$ e o desvio percentual médio $(\mathrm{P})$, calculado conforme a equação

$$
P=\frac{100}{n} \cdot \sum_{i=1}^{n} \frac{\left|\left(X_{\text {exp }}-X_{\text {pre }}\right)\right|}{X_{\text {exp }}}
$$

em que $\mathrm{P}=$ desvio percentual médio ( $\%) ; \mathrm{X}_{\text {exp }}=$ valores obtidos experimentalmente; $\mathrm{X}_{\text {pred }}=$ valores preditos pelo modelo; $\mathrm{n}=$ número de dados experimentais.

\section{RESULTADOS E DISCUSSÃO}

Com base nos dados apresentados nas Tabelas 1 e 2, observa-se que, pelos coeficientes de determinação, todos os modelos apresentaram ajustes de $99 \%$ aos dados experimentais, porém, para o juazeiro, todos os modelos apresentaram ajustes acima de $10 \%$, para o desvio percentual médio. Aguerre et al. (1989) consideram que resultados acima de $10 \%$ não indicam ajuste adequado dos valores observados, em relação aos ajustados pelos modelos em questão.

Para o mororó, somente o modelo de Oswin apresentou desvio percentual médio acima de $10 \%$, o que permite indicar que os modelos de GAB e Peleg são os que melhor descrevem o processo de dessorção das folhas apenas para mororó, às temperaturas de

Tabela 1. Parâmetros, coeficientes de determinação $\left(\mathrm{R}^{2}\right)$ e desvio percentual médio $(\mathrm{P})$ dos modelos ajustados à isoterma de dessorção das folhas de juazeiro (Campina Grande, PB, 2012).

\begin{tabular}{|c|c|c|c|c|c|c|c|}
\hline \multirow[t]{2}{*}{ Modelo } & \multirow[t]{2}{*}{ Temperatura } & \multicolumn{4}{|c|}{ Parâmetro } & \multirow[t]{2}{*}{$\mathrm{R}^{2}(\%)$} & \multirow[t]{2}{*}{$\mathrm{P}(\%)$} \\
\hline & & $\mathrm{Xm}$ & $\mathrm{C}$ & $\mathrm{K}$ & & & \\
\hline \multirow{3}{*}{ GAB } & $20^{\circ} \mathrm{C}$ & 4,3471 & 4,5212 & 0,9993 & & 98,76 & 17,2241 \\
\hline & $30^{\circ} \mathrm{C}$ & 6,0425 & 0,6014 & 0,9758 & & 99,88 & 14,0792 \\
\hline & $40^{\circ} \mathrm{C}$ & 4,9498 & 0,5397 & 0,9885 & & 99,88 & 15,0863 \\
\hline \multirow{4}{*}{ Oswin } & & $\mathrm{a}$ & & & $\mathrm{b}$ & & \\
\hline & $20^{\circ} \mathrm{C}$ & 6,7198 & & & 0,8961 & 98,46 & 17,5272 \\
\hline & $30^{\circ} \mathrm{C}$ & 5,0670 & & & 0,9328 & 99,72 & 17,7691 \\
\hline & $40^{\circ} \mathrm{C}$ & 4,0962 & & & 0,9846 & 99,79 & 15,4595 \\
\hline \multirow{4}{*}{ Peleg } & & $\mathrm{k}_{1}$ & $\mathrm{n}_{1}$ & $\mathrm{k}_{2}$ & $\mathrm{n}_{2}$ & & \\
\hline & $20^{\circ} \mathrm{C}$ & 124,24 & 50,4318 & 46,4343 & 2,5273 & 99,61 & 14,8810 \\
\hline & $30^{\circ} \mathrm{C}$ & 36,2231 & 3,5045 & 115,897 & 19,9438 & 99,95 & 15,5016 \\
\hline & $40^{\circ} \mathrm{C}$ & 34,1743 & 3,1867 & 142,442 & 23,1603 & 99,97 & 14,4862 \\
\hline
\end{tabular}

$\mathrm{X}_{\mathrm{m}}=$ umidade na monocamada molecular; $\mathrm{C}, \mathrm{K}$, a e $\mathrm{b}=$ parâmetros que dependem da temperatura e da natureza do produto; $\mathrm{K}_{1}, \mathrm{~K}_{2}, \mathrm{n}_{1}$ e $\mathrm{n}_{2}=$ constantes da equação.

Tabela 2. Parâmetros, coeficientes de determinação $\left(\mathrm{R}^{2}\right)$ e desvio percentual médio $(\mathrm{P})$ dos modelos ajustados à isoterma de dessorção das folhas de mororó (Campina Grande, PB, 2012).

\begin{tabular}{|c|c|c|c|c|c|c|c|}
\hline \multirow[t]{2}{*}{ Modelo } & \multirow[t]{2}{*}{ Temperatura } & \multicolumn{4}{|c|}{ Parâmetro } & \multirow[t]{2}{*}{$\mathrm{R}^{2}(\%)$} & \multirow[t]{2}{*}{$\mathrm{P}(\%)$} \\
\hline & & $\mathrm{Xm}$ & $\mathrm{C}$ & $\mathrm{K}$ & & & \\
\hline \multirow{3}{*}{ GAB } & $20^{\circ} \mathrm{C}$ & 14,5950 & 0,2275 & 0,9636 & & 99,64 & 8,1513 \\
\hline & $30^{\circ} \mathrm{C}$ & 16,120 & 0,3482 & 0,9849 & & 99,82 & 10,6963 \\
\hline & $40^{\circ} \mathrm{C}$ & 6,7217 & 0,4957 & 0,9981 & & 99,73 & 8,6689 \\
\hline \multirow{4}{*}{ Oswin } & & $\mathrm{a}$ & & & $\mathrm{b}$ & & \\
\hline & $20^{\circ} \mathrm{C}$ & 6,71659 & & & 1,02256 & 99,42 & 15,6738 \\
\hline & $30^{\circ} \mathrm{C}$ & 6,45931 & & & 0,97211 & 99,29 & 14,4843 \\
\hline & $40^{\circ} \mathrm{C}$ & 5,02374 & & & 1,07452 & 99,71 & 12,2861 \\
\hline \multirow{4}{*}{ Peleg } & & $\mathrm{k}_{1}$ & $\mathrm{n}_{1}$ & $\mathrm{k}_{2}$ & $\mathrm{n}_{2}$ & & \\
\hline & $20^{\circ} \mathrm{C}$ & 168,205 & 13,2892 & 20,4955 & 1,9401 & 99,71 & 6,8864 \\
\hline & $30^{\circ} \mathrm{C}$ & 189,857 & 15,2434 & 36,9384 & 2,6596 & 99,88 & 7,1331 \\
\hline & $40^{\circ} \mathrm{C}$ & 226,846 & 22,3592 & 48,4275 & 3,7883 & 99,62 & 8,8281 \\
\hline
\end{tabular}

$\mathrm{X}_{\mathrm{m}}=$ umidade na monocamada molecular; $\mathrm{C}, \mathrm{K}$, a e $\mathrm{b}=$ parâmetros que dependem da temperatura e da natureza do produto; $\mathrm{K}_{1}, \mathrm{~K}_{2}, \mathrm{n}_{1}$ e $\mathrm{n}_{2}=$ constantes da equação. 
$20^{\circ} \mathrm{C}, 30^{\circ} \mathrm{C}$ e $40^{\circ} \mathrm{C}$. Mohaptra \& Rao (2005) indicam boa adequação do modelo proposto para descrever o comportamento higroscópico.

Edoun et al. (2010), ao determinarem o teor de umidade de equilíbrio de folhas de eru (Gnetum africanum Wewl), para a faixa de temperatura de $30-50^{\circ} \mathrm{C}$ e umidade relativa de $10-90 \%$, verificaram que o modelo de GAB foi o que melhor descreveu as isotermas. Mohamed et al. (2004), ao estudarem o teor de umidade de equilíbrio de folhas de laranja (Citrus auratium L.), concluíram que, dentre os modelos testados, o de GAB e BET ajustaram-se satisfatoriamente aos dados experimentais de adsorção.

De acordo com os resultados apresentados nas Tabelas 1 e 2, verifica-se que, dentre os três modelos testados, o modelo de quatro parâmetros de Peleg destaca-se satisfatoriamente, na representação dos dados, por apresentar coeficientes de determinação superiores a $99 \%$ e os menores desvios médios. $\mathrm{O}$ modelo de Oswin, com dois parâmetros, que é empírico e baseia-se na expansão matemática para curvas de formato sigmoidal, apresentou ajustes inferiores, quando comparados aos demais modelos. Dessa forma, a equação de Peleg pode ser utilizada para estimar a representação higroscópica do fenômeno, nas condições em que o estudo foi desenvolvido.

Esses resultados estão de acordo com os encontrados por Bahloul et al. (2008), que, ao estudarem o comportamento higroscópico de adsorção e dessorção de quatro variedades de folhas de oliveira, em três temperaturas $\left(30^{\circ} \mathrm{C}, 40^{\circ} \mathrm{C}\right.$ e $\left.50^{\circ} \mathrm{C}\right)$, afirmaram que o modelo de Peleg forneceu o melhor ajuste aos dados experimentais, para a atividade de água: 0,058-0,898.

Silva et al. (2007) determinaram as isotermas de dessorção de Calendula officinalis L., em diferentes temperaturas $\left(30^{\circ} \mathrm{C}, 45^{\circ} \mathrm{C}\right.$ e $\left.60^{\circ} \mathrm{C}\right)$, por meio do uso de método gravimétrico, a fim de verificar a influência da temperatura na atividade de água. Os dados experimentais foram ajustados aos modelos matemáticos de BET, GAB, Halsey, Oswin, Peleg, Henderson e Chung Pfost. No entanto, Caetano et al. (2012) estudaram a higroscopicidade de sementes de caju-de-árvore-do-cerrado, e a equação que melhor se adequou aos dados foi a de Chung Pfost.

Silva et al. (2010), ao estudarem as características higroscópicas de folhas de coentro, observaram que as equações de Smith e Peleg apresentaram boa relação, quanto à descrição do comportamento dos dados experimentais da folha desidratada. No entanto, o modelo matemático de GAB apresentou desvios percentuais médios abaixo de $10 \%$, nas temperaturas de $20^{\circ} \mathrm{C}$ e $40^{\circ} \mathrm{C}(8,15 \%$ e $8,67 \%)$, e desvio percentual aceitável, na temperatura de $30^{\circ} \mathrm{C}(10,69 \%)$.

Observando-se os parâmetros do modelo de GAB (Tabelas 1 e 2), constatou-se que a umidade na monocamada molecular $(\mathrm{Xm})$ variou em torno de 4 a 16 (\% em base seca), entre as espécies em estudo. $\mathrm{O}$ conteúdo de umidade na monocamada $(\mathrm{Xm})$ é a quantidade de água que está fortemente adsorvida na superfície do produto, sendo considerado importante para o armazenamento por longos períodos.

Resultados semelhantes foram encontrados por Ghodake et al. (2007), estudando a higroscopicidade de folhas de chá preto e chá verde, nas temperaturas de $20^{\circ} \mathrm{C}, 30^{\circ} \mathrm{C}$ e $40^{\circ} \mathrm{C}$ e umidade relativa de $10-90 \%$, e que, ao ajustarem o modelo de $\mathrm{GAB}$ às isotermas de sorção, encontraram valores de Xm variando de 6 a 12.

Verificou-se, também, por meio das Tabelas 1 e 2, que, para o modelo de GAB, os valores de $\mathrm{k}$ apresentam-se menores que 1 , variando entre $0,96 \mathrm{e}$ 0,99 e não apresentando comportamento definido, em relação às temperaturas estudadas.

$\mathrm{O}$ parâmetro $\mathrm{C}$, para a espécie mororó, demonstrou comportamento inversamente proporcional ao aumento da temperatura. Já para o juazeiro, esse valor oscilou de 0,5 a 4,5. Observou-se que o parâmetro $\mathrm{C}$, em ambas as espécies, mostrou-se inferior a 200, encontrando-se dentro da faixa comumente registrada na literatura (Alexandre et al. 2007).

O parâmetro K representa o fator de correção das propriedades das moléculas na multicamada, com relação ao volume de líquido, enquanto a constante de sorção $C$ é função das interações entre os sítios ativos do produto e as moléculas de água (Alexandre et al. 2007).

Nos ajustes com o modelo de Peleg, as constantes $\mathrm{k}_{1}, \mathrm{n}_{1}, \mathrm{k}_{2}$ e $\mathrm{n}_{2}$ apresentaram comportamentos diferentes, entre as espécies. Para o juazeiro, houve discrepância entre os valores (Tabela 1). Verificou-se que os parâmetros $\mathrm{k}_{1}$ e $\mathrm{n}_{1}$ diminuíram com o aumento da temperatura, sendo inversamente proporcionais a $\mathrm{k}_{2}$ e $\mathrm{n}_{2}$. Já para o mororó, $\mathrm{k}_{1}, \mathrm{n}_{1}, \mathrm{k}_{2}$ e $\mathrm{n}_{2}$ apresentaram tendência de acréscimo, com o aumento da temperatura.

Silva et al. (2007) verificaram que, para Calendula officinalis L., os parâmetros $\mathrm{k}_{1}$ e $\mathrm{n}_{1}$ do modelo de Peleg apresentaram decréscimo em seus valores, com o aumento da temperatura. Com relação aos demais parâmetros, a temperatura de $45^{\circ} \mathrm{C}$ foi levemente superior que a de $60^{\circ} \mathrm{C}$. Park et 
al. (2002), em estudo dos parâmetros de secagem e isotermas de dessorção em folhas de menta (Mentha crispa L.) desidratadas, em temperaturas de $30^{\circ} \mathrm{C}$ e $40^{\circ} \mathrm{C}$, constataram que o modelo de Peleg foi o que apresentou menor erro relativo $(1,82 \%$ e $3,82 \%$, respectivamente).

Analisando-se os ajustes, pelo modelo de Oswin, constatou-se que, para o juazeiro, os parâmetros $a$ e $b$ apresentaram tendência de acréscimo, com o aumento da temperatura. Já para as folhas de mororó, observou-se que as constantes $a$ e $b$, na temperatura de $30^{\circ} \mathrm{C}$, apresentaram tendência de decréscimo, com o aumento da temperatura. Park et al. (2001), estudando o comportamento das isotermas de sorção de pêra bartlett (Pyrus sp.) in natura e desidratada osmoticamente, utilizando sais e três temperaturas $\left(40^{\circ} \mathrm{C}, 60^{\circ} \mathrm{C}\right.$ e $\left.80^{\circ} \mathrm{C}\right)$, obtiveram, com o modelo de Oswin e com o aumento da temperatura, acréscimo da constante $b$, com um pequeno decréscimo na temperatura de $80^{\circ} \mathrm{C}$ e diminuição da constante $a$.

$\mathrm{Na}$ Figura 1 são apresentadas as isotermas de dessorção de umidade das folhas in natura de juazeiro, em três temperaturas $\left(20^{\circ} \mathrm{C}, 30^{\circ} \mathrm{C}\right.$ e $\left.40^{\circ} \mathrm{C}\right)$, ajustadas pelo modelo de Peleg. Verificou-se que as isotermas apresentaram formato sigmoidal, típico de produtos com alto teor de água.

Observou-se que, quando a temperatura aumenta, o conteúdo de umidade de equilíbrio decresce para o mesmo valor de atividade de água. Esse comportamento, segundo Lahsasni et al. (2003), é atribuído ao alto estado de excitação das moléculas de água, em temperaturas elevadas, diminuindo as forças de atração entre as moléculas. Esses resultados corroboram os obtidos para produtos agrícolas (Resende et al. 2006, Gonelli 2008, Ferreira et al. 2011).

Verificou-se, também, que a umidade de equilíbrio cresce com o aumento da atividade de água, para todas as temperaturas. Esses resultados estão de acordo com os encontrados por Silva et al. (2010), ao estudarem as características higroscópica e termodinâmica do coentro desidratado. Observou-se que, para a temperatura de $20^{\circ} \mathrm{C}$, em atividades de água menores que 0,5, as curvas apresentaram maiores desvios, em relação aos dados experimentais. Para atividades de água compreendidas entre 0,9 e 0,95, todas as curvas possuem comportamentos similares.

A Figura 2 exibe a representação gráfica do modelo de Peleg, aplicado aos dados experimentais das isotermas de dessorção das folhas de mororó, nas temperaturas de $20^{\circ} \mathrm{C}, 30^{\circ} \mathrm{C}$ e $40^{\circ} \mathrm{C}$. Contatou-se que as isotermas também apresentaram formato sigmoidal, característico de curvas do tipo II, de acordo com a classificação de Brunauer et al. (1938), que considera o formato sigmoidal do equilíbrio higroscópico padrão para produtos agrícolas.

Observou-se que não existe grande influência da temperatura sobre as umidades de equilíbrio das folhas in natura, para atividade de água inferior a 0,3 . A partir de 0,6 , a curva referente à temperatura de $20^{\circ} \mathrm{C}$ ocupa posição superior, em relação às demais temperaturas. No entanto, a curva de $30^{\circ} \mathrm{C}$ encontra-se em posição inferior à de $40^{\circ} \mathrm{C}$, em atividade de água de 0,7 até, aproximadamente, 0,8 , sobrepondo-se na atividade de água de 0,9.

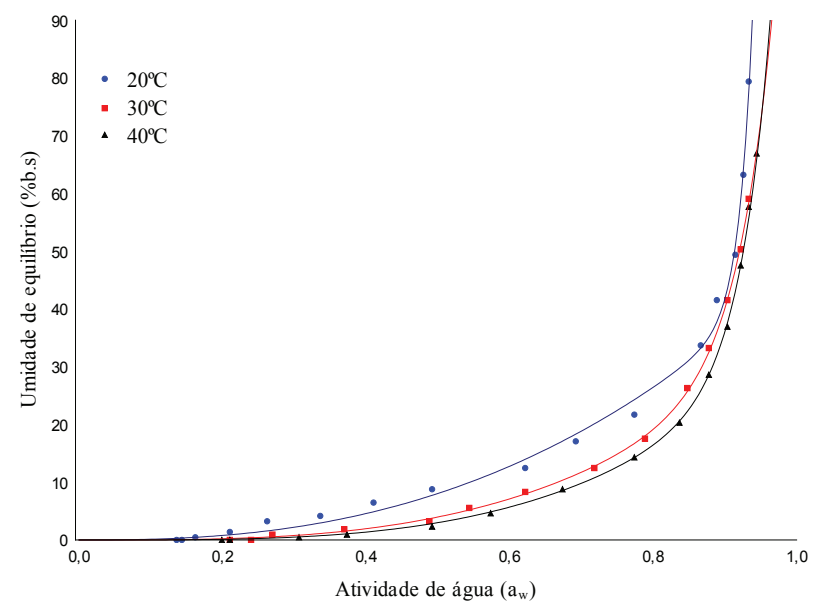

Figura 1. Isotermas de dessorção de folhas in natura de juazeiro ajustadas pelo modelo de Peleg (Campina Grande, $\mathrm{PB}, 2012)$.

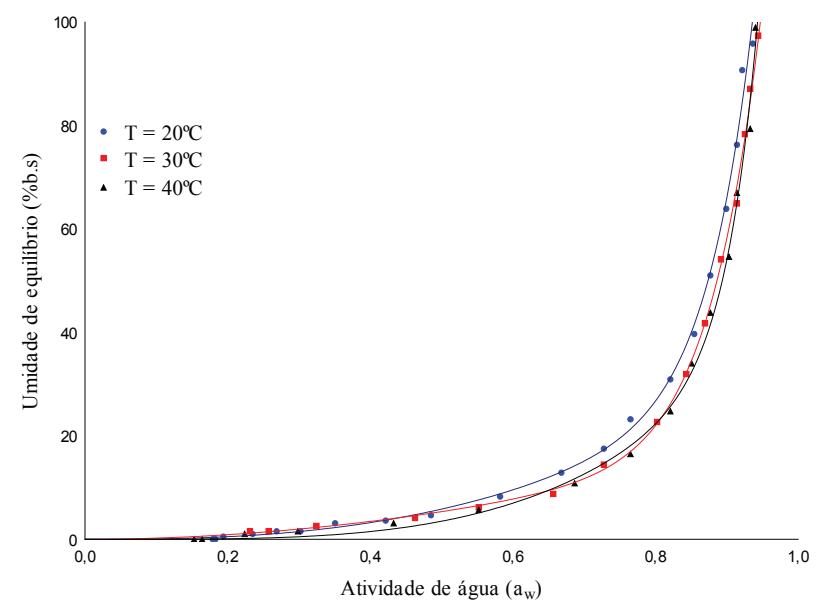

Figura 2. Isotermas de dessorção de folhas in natura de mororó ajustadas pelo modelo de Peleg (Campina Grande, PB, 2012). 
O valor do teor de umidade de equilíbrio, para diversos materiais, depende da maneira como a condição de equilíbrio é atingida. Um determinado valor é obtido quando o produto úmido perde umidade por dessorção, durante a secagem (Rocha 2011). Em geral, a umidade de equilíbrio diminui com o aumento da temperatura. Essa diminuição pode ser justificada com base no aumento da pressão do vapor de água no ar da superfície do produto, por apresentar maior número de moléculas de água que o ar (Oliveira et al. 2006).

O conteúdo de equilíbrio de umidade avalia a perda ou o ganho de água e está relacionado diretamente com os processos de secagem e armazenagem de produtos agrícolas. O método de conservação utilizando a secagem baseia-se principalmente na diminuição do teor de água disponível no material vegetal e, consequentemente, serão reduzidas as atividades enzimática, química e biológica, garantindo armazenamento seguro.

\section{CONCLUSÕES}

1. As isotermas obtidas apresentaram formato sigmoidal, padrão para o equilíbrio higroscópico de produtos agrícolas.

2. O modelo que melhor representou as isotermas de dessorção das folhas de juazeiro e mororó, na faixa de temperatura de $20-40^{\circ} \mathrm{C}$, foi o de Peleg.

\section{REFERÊNCIAS}

AGRA, M. F. et al. Medicinal and poisonous diversity of the flora of "Cariri Paraibano" Brazil. Journal of Ethnopharmacology, Escarpa, v. 2, n. 111, p. 383-395, 2007.

AGUERRE, R. J.; SUAREZ, C.; VIOLLAZ, P. E. New BET type multi-layer sorption isotherms: part II. Modeling water sorption in foods. Lebensmittel-wissenschaft und Technologie, Londres, v. 22, n. 4, p. 192-195, 1989.

ALEXANDRE, H. V.; FIGUEIRÊDO, R. M. F.; QUEIROZ, A. J. M. Isotermas de adsorção de umidade da pitanga em pó. Revista de Biologia e Ciências da Terra, Campina Grande, v. 7, n. 1, p. 11-20, 2007.

BAHLOUL, N.; BOUDHRIOUA, N.; KECHAOU, N. Moisture desorption-adsorption isotherms and isosteric Nheats of sorption of Tunisian olive leaves (Olea europaea L.). Industrial Crops and Products, Peoria, v. 28, n. 2, p. 162-176, 2008.

BRUNAUER, S.; EMMETT, P. H.; TELLER, E. Adsorption of gases in multimolecular layers. Journal of the American Chemical Society, Nova Iorque, v. 60, n. 2, p. 309-319, 1938.

CAETANO, G. S. et al. Higroscopicidade de sementes de caju-de-árvore-do-cerrado. Pesquisa Agropecuária Tropical, Goiânia, v. 42, n. 4, p. 437-445, 2012.

CAPRISTE, G. H.; ROTSTEIN, E. Prediction of sorptional equilibrium data for starch-containing foodstuffs. Journal of Food Science, Chicago, v. 47, n. 8, p. 1501-1507, 1982.

COSTA, C. M. L. Caracterização e análise experimental do recobrimento de sementes de jambu (Spilhantes oleracea) em leito fluidizado. 2010. $203 \mathrm{f}$. Tese (Doutorado em Engenharia Química) - Faculdade de Engenharia Química, Universidade Estadual de Campinas, Campinas, 2010.

EDOUN, M.; KUITCHE, A.; KAPSEU, C. Determination of the Gnetum africanum water sorption isotherms for use in the design of an adapted dryer. International Journal of Food Science and Technology, Rio de Janeiro, v. 45, n. 1, p. 105-112, 2010.

FERREIRA, S. C. S.; SILVA, H. W.; RODOVALHO, R. S. Isoterma de dessorção e calor latente de vaporização da semente de pimenta cumari amarela (Capsicum chinense L.). Revista Liberato, Nova Hamburgo, v. 12, n. 18, p. 107-206, 2011.

GHODAKE, H. M.; GOSWAMI, T. K.; CHAKRAVERTY, A. Moisture sorption isotherms, heat of sorption and vaporization of withered leaves, black and green tea. Journal of Food Engineering, Ontário, v. 78, n. 3, p. 827835, 2007.

GONELLI, A. L. D. Variação das propriedades físicomecânicas e da qualidade da mamona (Ricinus communis L.) durante a secagem e o armazenamento. 2008. $186 \mathrm{f}$. Tese (Doutorado em Engenharia Agrícola) - Universidade Federal de Viçosa, Viçosa, 2008.

LAHSASNI, S. et al. Moisture adsorption-desorption isotherms of prickly pear cladode (Opuntia ficus indica) at different temperatures. Energy Conversion \& Management, Marrakech, v. 44, n. 6, p. 923-936, 2003.

MOHAMED, L. A. et al. Moisture sorption isotherms and heat of sorption of bitter orange leaves (Citrus aurantium). Journal of Food Engineering, Ontário, v. 67, n. 4, p. 491498, 2004.

MOHAPATRA, D.; RAO, P. S. A thin layer drying model of parboiled wheat. Journal of Food Engineering, Ontário, v. 66, n. 4, p. $513-518,2005$.

OLIVEIRA, M. M. et al. Isotermas de dessorção da casca do maracujá (Passiflora edulis Sims): determinação experimental e avaliação de modelos matemáticos. Ciência Rural, Santa Maria, v. 36, n. 5, p. 1624-1629, 2006. 
PARK, K. J.; BIN, A.; BROD, F. P. R. Obtenção das isotermas de sorção e modelagem matemática para a pêra bartlett (Pyrus sp.) com e sem desidratação osmótica. Ciência e Tecnologia de Alimentos, Campinas, v. 21, n. 1, p. 73-77, 2001.

PARK, K. J.; VOHNIKOVA, Z.; BROD, F. P. R. Evaluation of drying parameters and desorption isotherms of garden mint leaves (Mentha crispa L.). Journal of Food Engineering, Ontário, v. 51, n. 3, p. 193-199, 2002.

RESENDE, O. et al. Isotermas e calor isostérico de sorção do feijão. Ciência e Tecnologia de Alimentos, Campinas, v. 26, n. 3, p. 626-631, 2006.

ROCHA, R. P. Avaliação do teor e da composição do óleo essencial de Cymbopogon citratus e Thymus vulgaris submetidos a processos de secagem e armazenamento. 2011. 149 f. Tese (Doutorado em Engenharia Agrícola) Universidade Federal de Viçosa, Viçosa, 2011.

SILVA, A. S. A. et al. Característica higroscópica e termodinâmica do coentro desidratado. Revista Ciência Agronômica, Fortaleza, v. 41, n. 2, p. 237-244, 2010.

SILVA, F.; PARK, K. J.; MAGALHÃES, P. M. Isotermas de dessorção de Calendula officinalis L.: determinação experimental e modelagem matemática. Revista Brasileira de Plantas Medicinais, Botucatu, v. 9, n. 1, p. 21-28, 2007.

SOUSA, F. C. et al. Propriedades físicas e físico-químicas da polpa de juazeiro. Revista Verde de Agroecologia e Desenvolvimento Sustentável, Mossoró, v. 8, n. 2, p. 6871, 2013. 